\title{
Criminologie
}

\section{Les échecs des politiques antiterroristes russes au Caucase du Nord}

\section{Aurélie Campana}

Volume 47, numéro 2, automne 2014

Criminalité et police transnationales : une perspective critique

URI : https://id.erudit.org/iderudit/1026727ar

DOI : https://doi.org/10.7202/1026727ar

Aller au sommaire du numéro

\section{Éditeur(s)}

Les Presses de l’Université de Montréal

ISSN

0316-0041 (imprimé)

1492-1367 (numérique)

Découvrir la revue

Citer cet article

Campana, A. (2014). Les échecs des politiques antiterroristes russes au Caucase du Nord. Criminologie, 47(2), 35-56. https://doi.org/10.7202/1026727ar
Résumé de l'article

Cet article porte sur les dynamiques propres aux politiques russes antiterroristes au Caucase du Nord et analyse leurs impacts sur le conflit. Pour ce faire, il s'appuie sur le concept de configuration tel que développé par $\mathrm{N}$. Elias. Il se propose de déconstruire les interdépendances qui lient les acteurs de l'antiterrorisme et d'en examiner la nature et les logiques. Il montre qu'il existe non seulement un décalage entre les discours et les pratiques, mais également une divergence d'intérêts et de croyances, que la prédominance du clanisme, du localisme et du clientélisme, comme modes d'interactions et principes organisationnels, ne fait qu'enraciner. Il explique les échecs des politiques antiterroristes par les jeux de pouvoir, qui à Moscou et au Nord-Caucase, entravent leur bonne mise en oeuvre, par l'absence de coordination et la compétition inter-agences et par le détournement de la violence à des fins privées. Il montre ainsi que loin de contenir le conflit, ces pratiques imputables aux différents acteurs impliqués dans la lutte anti-terroriste participent à la montée des violences et entretiennent un conflit aux logiques multiples. 


\title{
Les échecs des politiques antiterroristes russes au Caucase du Nord
}

\author{
Aurélie Campana ${ }^{1}$ \\ Professeure agrégée \\ Département de science politique \\ Université Laval \\ aurelie.campana@pol.ulaval.ca
}

\begin{abstract}
RÉSUMÉ - Cet article porte sur les dynamiques propres aux politiques russes antiterroristes au Caucase du Nord et analyse leurs impacts sur le conflit. Pour ce faire, il s'appuie sur le concept de configuration tel que développé par N. Elias. Il se propose de déconstruire les interdépendances qui lient les acteurs de l'antiterrorisme et d'en examiner la nature et les logiques. Il montre qu'il existe non seulement un décalage entre les discours et les pratiques, mais également une divergence d'intérêts et de croyances, que la prédominance du clanisme, du localisme et du clientélisme, comme modes d'interactions et principes organisationnels, ne fait qu'enraciner. Il explique les échecs des politiques antiterroristes par les jeux de pouvoir, qui à Moscou et au NordCaucase, entravent leur bonne mise en cuvre, par l'absence de coordination et la compétition inter-agences et par le détournement de la violence à des fins privées. Il montre ainsi que loin de contenir le conflit, ces pratiques imputables aux différents acteurs impliqués dans la lutte anti-terroriste participent à la montée des violences et entretiennent un conflit aux logiques multiples.
\end{abstract}

MotS-CLÉs - Russie, Caucase du Nord, politiques antiterroristes, réseaux, interdépendances.

\section{Introduction}

Le Caucase du Nord, région située à la frontière sud de la Fédération de Russie, est secoué depuis le milieu des années 1990 par des violences

1. Université Laval, Département de science politique, Pavillon de Koninck, 1030, avenue des Sciences-Humaines, Local 4423, Québec, (Québec), Canada, G1V 0A6. 
récurrentes. Les deux guerres ${ }^{2}$ russo-tchétchènes (1994-1996 et 19992009) en constituent les épisodes aux conséquences les plus durables (Gilligan, 2009; Sakwa, 2005; Schaefer, 2011; Tishkov, 2004; Wood, 2007). En 2009, le président russe alors en poste, Dmitri Medvedev, déclare la fin de «l'opération antiterroriste» que les forces armées russes ont lancée en 1999, mettant officiellement un terme à un conflit, qui reste pourtant non résolu. D'une part, des violences continuent régulièrement à secouer la Tchétchénie. D'autre part, aucune négociation politique n'a été entreprise, les dirigeants russes ayant privilégié une stratégie de «tchétchénisation» du conflit (Russell, 2008). Cette dernière a conduit à la mise en place d'un régime tchétchène prorusse autoritaire (Russell, 2010). Surtout, les dirigeants russes ont choisi d'occulter jusqu'en 2009-2010 une réalité tangible depuis 2003: la montée des violences dans les républiques voisines de la Tchétchénie, évolution qui consacre la diffusion progressive du conflit tchétchène au-delà des frontières de la république. Alors que l'épicentre des violences se situe encore en Tchétchénie en 2006, il se déplace petit à petit vers l'Ingouchie et le Daghestan. En 2013, le Daghestan constitue la république la plus instable du Caucase du Nord. Bien que le nombre des violences ait diminué en Ingouchie depuis 2009, l'équilibre qui y règne demeure fragile. Enfin, la Kabardino-Balkarie a gagné en instabilité depuis 2010 (Dzutsev, 2010a; Schwartzbaum, 2013; Vatchagaev, 2011).

Les travaux portant sur cette région ont principalement abordé ce phénomène de régionalisation de la violence sous quatre angles non exclusifs. Le premier souligne les conséquences de la seconde guerre russo-tchétchène (O'Loughlin \& Witmer, 2011). Le second insiste sur la nature islamiste de l'insurrection, qui s'est développée sur fond de réislamisation des sociétés nord-caucasiennes et de progression du salafisme (Malashenko \& Yarlykapov, 2009; Shterin \& Yarlykapov, 2011). Le troisième attribue cette montée de la violence aux difficultés structurelles que connaît cette partie de la Russie qui compte parmi les plus pauvres de la Fédération (Sokolov, Magomegov \& Silaev, 2013). Le quatrième fait de la répression indiscriminée un facteur de la radicalisation de nombre de jeunes Nord-Caucasiens (Toft \& Zhukov, 2012).

2. Les dirigeants russes ont refusé de qualifier la seconde intervention en Tchétchénie de guerre, préférant utiliser le terme «opération antiterroriste». 
Si ces explications saisissent une partie des processus à l'œuvre, elles tendent à se concentrer avant tout sur l'environnement socioéconomique, politique et religieux, ainsi que sur les stratégies des insurgés. Peu de travaux portent sur les dynamiques propres aux politiques russes antiterroristes et leurs impacts sur le conflit. Pourtant, sans en faire une cause directe de l'expansion géographique du conflit, nous faisons l'hypothèse que les réponses formulées par l'État russe participent des processus qui nourrissent la montée de la violence dans cette région déjà profondément instable. Cet article aborde cet aspect précis. Il traite plus particulièrement des interactions entre les différents acteurs impliqués dans la mise en œuvre des politiques décidées à Moscou. Ce faisant, il met en évidence un décalage patent entre discours et pratiques ainsi que l'existence de croyances, d'intérêts et des temporalités divergents. Il analyse les impensés et les impasses de la «politique antiterroriste russe», telle que dessinée sous Dmitri Medvedev (2008-2012). Cette dernière a été, du moins sur papier, réorientée $e^{3}$. Après avoir privilégié une approche purement coercitive, Moscou s'est engagé dans une stratégie qui allie répression, contrôle des dirigeants locaux et tentative de gagner l'adhésion des populations locales (Campana, 2013b). L'objectif est de tenter de contenir le conflit et d'éviter que la persistance de ce dernier ne vienne perturber la bonne tenue des Jeux olympiques de Sotchi, qui se déroulent à une encablure des républiques les plus instables.

Cet article comporte trois parties. La première présente notre cadre analytique, basé sur le concept de configuration violente, qui met en évidence le rôle des interactions entre acteurs et des interdépendances qui les lient dans les dynamiques de violences. La seconde partie revient brièvement sur l'historique des conflits dans cette région, sur les réponses formulées par l'État russe et sur leurs évolutions. La troisième partie s'arrête sur les interdépendances entre les différents acteurs de l'antiterrorisme et sur leur nature. Ces dernières, qui mêlent coopération, compétition et affrontement direct ou indirect, ne peuvent se

3. Une telle approche a été mise en œuvre en 2009, mais plusieurs de ses prémisses ont été timidement développées après la prise d'otages de Beslan en 2004 et le constat de la nécessité de mettre sur pied un plan de développement socioéconomique pour la région (Melvin, 2007, p. 52). Le représentant du président de la Fédération dans le district fédéral du Sud alors en poste, Dmitri Kozak, a joué un rôle central dans la promotion d'une telle approche. Il a toutefois fallu attendre l'annonce de la fin de l'opération antiterroriste en Tchétchénie en 2009 pour qu'elle soit véritablement déployée. 
comprendre sans tenir compte du localisme, du clientélisme et du clanisme qui agissent comme autant de principes organisateurs sur le plan local. Nous montrons que de telles pratiques contribuent à alimenter le cycle des violences, entravant les politiques antiterroristes et contreinsurrectionnelles jusqu'à créer les conditions de l'échec.

\section{Le conflit comme configuration violente}

Nous postulons que la violence relève de processus relationnels (Tilly, 2005). Nous distinguons quatre relations idéal-typiques: entre les acteurs engagés dans un jeu de nature conflictuelle; entre les acteurs qui appartiennent à une même mouvance ou à une même catégorie d'acteurs, insurgés et acteurs étatiques par exemple; entre ces différentes catégories d'acteurs et l'espace social et politique dans lequel ils évoluent; et entre ces mêmes acteurs et les environnements locaux, régionaux et internationaux. Dans le cadre de cet article, nous nous concentrerons sur le second idéaltype, sans toutefois écarter les autres types de relations, en tant qu'elles influencent la nature et la densité des interactions entre les groupes et les individus qui appartiennent à la catégorie «acteurs étatiques».

Une telle approche oblige à déconstruire la catégorie «acteurs étatiques», suivant la perspective défendue par le courant des "microdynamiques des conflits», qui montrent que les conflits internes ${ }^{4}$ aux États ne sont pas des «binary conflicts but complex and ambiguous processes that foster an apparently massive, though variable, mix of identities and actions» (Kalyvas, 2003, p. 475). Elle conduit également à considérer les interdépendances qui lient les différents acteurs en conflit. Afin de mieux saisir ce concept d'interdépendances et sa contribution à une meilleure compréhension des dynamiques conflictuelles, nous proposons d'utiliser le concept de configuration tel que développé par Norbert Elias. Selon le sociologue allemand, une configuration

4. Les travaux de Kalyvas portent sur les guerres civiles. Les conclusions des auteurs qui s'inscrivent dans ce courant s'avèrent toutefois transposables à tous les types de conflits internes aux États. Nous ne souhaitons pas rentrer dans les débats définitionnels qui entourent les concepts de guerre civile. Cette entreprise dépasse en effet l'objectif de cet article. Nous partirons toutefois du postulat que les conflits qui se déroulent au Caucase du Nord appartiennent à la catégorie des guerres civiles asymétriques dites «irrégulières», en ce qu'ils opposent des acteurs non étatiques divers à un État dont ils contestent l'autorité et en ce qu'ils se déroulent à la périphérie de ce même État (à cet effet, voir la catégorisation et la définition proposée par Kalyvas, 2007). 
existe quand des individus ou des groupes établissent des relations entre eux et rentrent en interdépendances (Elias, 1978, p. 130). Opposé à toute forme de réification des relations sociales, Elias (1978) souligne le caractère dynamique de la chaîne d'interdépendances qui se crée. Ces interdépendances lient les individus ou groupes appartenant à une même catégorie d'acteurs, mais également ceux qui appartiennent à des groupes opposés dans et par le conflit. La violence devenant un mode de dialogue dans un conflit intra-étatique, nous qualifions la configuration que nous étudions de configuration violente.

La transposition du concept de configuration à l'étude des conflits offre selon nous des pistes de réflexion intéressantes. En effet, ce concept met l'accent sur l'interpénétration dynamique des comportements, des représentations et des pratiques des différents acteurs impliqués dans le conflit. Les acteurs prennent des décisions stratégiques motivées par un ensemble de croyances et d'intérêts, mais contraintes par les comportements des autres acteurs (Elias \& Dunning, 1986). Les types d'interdépendances oscillent entre la coopération, l'alliance ou au contraire l'affrontement; ils incluent, comme le montrent de nombreuses recherches sur la violence politique, la surenchère ou la compétition (voir par exemple, Bloom, 2004; Devotta, 2005; Horowitz, 1985). Ces dynamiques relationnelles marquent de leur empreinte la chaîne d'interdépendances qui se crée et obligent les acteurs à de constants réajustements. Leur capacité à se repositionner dans un contexte mouvant dépend alors des ressources qu'ils possèdent, mais aussi de leur aptitude à jouer le jeu. Ceci veut dire que même un acteur initialement dépourvu de ressources matérielles ou symboliques peut progressivement gagner en influence et contribuer à modifier les règles du jeu.

Le concept de configuration invite à déconstruire les catégories d'acteurs et à analyser les interdépendances qui les lient, indépendamment des ressources et identités revendiquées avant le début du conflit. Il permet donc de déplacer le regard vers les clivages multiples, les identités à géométrie variable et les pratiques situées qui évoluent dans le conflit. Une telle perspective répond à l'une des critiques formulées à l'endroit des chercheurs qui s'inscrivent dans le courant des «microdynamiques» des conflits. Ces derniers se sont vu reprocher leur tendance à analyser le comportement des acteurs au regard des conditions qui prévalaient avant le début du conflit et sans toujours tenir compte de leurs évolutions une fois le conflit déclenché (Kalyvas, 2008, p. 403). De plus, une telle perspective permet de mieux penser les 
«interconnexions entre les différents niveaux d'analyse» (Kalyvas, 2010, p. xii), en tant que la notion de configuration offre «un continuum entre le macro et le micro» (Emirbayer, 1997, p. 294). Enfin, le nombre d'acteurs impliqués dans ce type de conflits rend, comme le rappelle Soeters (2005, p. 31), le jeu imprévisible et amoindrit la potentialité de lier le résultat aux préférences d'un acteur. Le concept de configuration permet ici de replacer l'ambiguïté au centre de l'analyse, en la considérant non pas comme «un bruit», mais comme «la structure même de ces conflits» (Raleigh, 2011, p. 473).

Avant d'analyser les différents acteurs impliqués et les dynamiques relationnelles auxquelles ils participent, nous faisons un bref historique des conflits qui secouent cette région et des réponses formulées par Moscou pour les circonscrire et ultimement y mettre fin.

\section{Le conflit au Caucase du Nord: bref rappel historique}

Un conflit, des conflits?

La montée des violences que connaît le Caucase du Nord depuis 2003 se situe dans le prolongement du second conflit russo-tchétchène. Plusieurs chefs de guerre tchétchènes ont mis en place une stratégie de diffusion du conflit, s'appuyant sur les réseaux interpersonnels tissés au cours des deux guerres et bénéficiant du soutien de mercenaires djihadistes (Moore \& Tumelty, 2008). Elle s'est concrétisée par la création, en 2007, de l'Émirat du Caucase du Nord, chargé de fédérer les différents groupes insurgés islamistes actifs dans la région (Hahn, 2011). Lapparition de cette structure aux contours flous consacre deux évolutions: le glissement de plusieurs groupes tchétchènes d'un registre nationaliste vers un registre islamiste et l'influence grandissante des non-Tchétchènes (Dagestanais, Ingouches et autres) au sein d'une insurrection devenue régionale (Campana \& Ratelle, 2014). La seconde guerre russo-tchétchène a eu d'autres impacts déstabilisateurs à plus ou moins long terme. Elle a drainé son lot de réfugiés, rentrés depuis en Tchétchénie, souvent sous la contrainte (Gilligan, 2009, pp. 98-118) et a constitué un terreau propice au développement de réseaux criminalisés (Galeotti, 2002). Elle a enfin entraîné une forte militarisation de la région (Melvin, 2007, p. 24). 
Si les conséquences de cette guerre ne doivent pas être minimisées, elles ne doivent en rien occulter les difficultés structurelles que traversent ces républiques. La région est en effet traversée de trois crises qui se superposent (Campana, 2013a). La première, d'ordre socioéconomique, engendre de graves inégalités, alimentées par un chômage galopant et une économie souterraine surdimensionnée par rapport à l'économie réelle. La seconde renvoie à la perte de légitimité des autorités locales, accusées de néo-patrionalisme et clanisme 5 (Derluguian, 2005). La troisième crise touche l'Islam traditionnel, engagé dans une compétition féroce avec les salafistes dans un contexte de réislamisation des sociétés nord-caucasiennes. Parallèlement, plusieurs des conflits qui ont secoué cette région dans les années 1990, que ces derniers aient été motivés par des enjeux politiques, territoriaux, mémoriels ou par l'accès aux ressources légales ou illégales, restent non résolus. Ils constituent autant de zones d'instabilité potentielle, dans un contexte de prolifération de groupes armés non démobilisés et de circulation massive d'armes («V Kabardino-Balkarii...», 2010). La méfiance envers l'État a largement été exploitée par ces groupes dont la nature et les motivations restent diverses. Ainsi, si les violences qui secouent le NordCaucase sont le plus souvent attribuées à des insurgés islamistes opposés à l'État, elles relèvent bien souvent d'autres logiques.

\section{Les stratégies russes entre antiterrorisme et contre-insurrection}

Les réponses russes à cette montée de la violence oscillent entre antiterrorisme et contre-insurrection, bien que le second terme ne soit jamais utilisé par les dirigeants russes. Cette posture rhétorique tient tant à la définition du problème qu'à une volonté explicite de criminaliser les opposants à l'État ${ }^{6}$ et de nier les dimensions politiques du conflit (Campana, 2014). Elle fait en cela suite aux stratégies, tant discursives que politiques et militaires, déployées lors du second conflit russo-tchétchène (Campana \& Légaré, 2011). L'antiterrorisme russe

5. Le clan est ici défini à la fois dans son acceptation anthropologique et comme un groupe uni par des intérêts politiques et/ou économiques. Les deux réalités se superposent en effet dans nombre de régions du Caucase du Nord.

6. La loi antiterroriste russe, telle qu'adoptée en 2006, définit le terrorisme comme une menace dirigée contre l'État, et non contre des civils. La loi de 2006 consacre ainsi une évolution par rapport à la législation antérieure (Soldatov \& Borogan, 2010, p. 174). 
privilégie une approche coercitive et répressive. Toutefois, à cette utilisation de la violence, souvent entachée d'abus (Amnesty International, 2012), s'articule depuis 2009-2010 une approche civile dont les objectifs sont de moderniser une région aux prises avec de multiples difficultés structurelles, de restaurer les capacités de l'État et de le doter d'une légitimité qui lui fait défaut pour ultimement couper les insurgés de leur base sociale. Cette dernière relève à bien des égards de la contreinsurrection, même si l'objectif n'est pas tant de «gagner les cœurs et les esprits» que d'assurer la loyauté des autorités et des populations locales envers Moscou. Cette approche se concrétise également par le financement de projets de développement économique (Markedonov, 2010). Une grande partie des subsides envoyés par Moscou viennent toutefois alimenter la corruption, considérée comme «endémique» dans la région («Korrupciâ na Severnom Kavkaze...», 2010).

Les acteurs chargés des différents aspects de l'intervention de l'État russe dans la région sont divers. Le président de la Fédération de Russie et son administration y tiennent un rôle premier. Entre 2008 et 2012, le premier ministre en fonction, Vladimir Poutine, a occupé une place centrale dans les processus de décision. Cet investissement tient à une redistribution informelle des pouvoirs et des rôles entre les deux têtes de l'exécutif sous Medvedev (Kryshtanovskaya, 2012, p. 7). Le représentant du président de la Fédération de Russie dans le district fédéral du Nord-Caucase, fonction créée en 2010, sert de courroie de transmission entre l'administration présidentielle fédérale et la région. Son titulaire depuis 2010, Aleksandr Khloponin, est supposément chargé de toutes les affaires régionales et doit être tenu informé des opérations antiterroristes, coordonnées depuis 2006 par le Comité national antiterroriste (NAK). Ce dernier est dirigé par le Service fédéral de sécurité (FSB), censé travailler de concert avec les troupes des ministères de l'Intérieur et de la Défense. Les ministères fédéraux des Régions, des Finances et du Développement économique sont également impliqués à différents niveaux. Ces acteurs fédéraux doivent parallèlement composer avec une multitude d'acteurs locaux: les présidents ou gouverneurs de chacune des entités fédérées qui composent le district fédéral du Caucase du Nord (les républiques de Tchétchénie, d'Ingouchie, du Daghestan, de Kabardino-Balkarie, de Karachevo-Tcherkessie, d'Ossétie du Nord et le territoire de Stavropol); les membres des gouvernements locaux; les polices et milices locales; sans oublier les représentants des sections locales du FSB, des ministères fédéraux de l'Intérieur et de 
la Défense, des renseignements militaires... pour ne nommer que les acteurs parmi les plus importants.

Si ces acteurs semblent partager une même appréciation de la situation dans la région, ils sont mus par des croyances et des intérêts parfois divergents. Dès lors, les interdépendances qui les lient se révèlent d'autant plus changeantes qu'elles sont organisées autour de trois principes, le localisme, le clanisme et le clientélisme.

\section{Des interdépendances à géométrie variable et l'impossible gestion de l'incertitude}

La configuration violente à laquelle les protagonistes de l'antiterrorisme russe participent implique une multitude d'acteurs, très divers par leur nature, leurs motivations et la structuration des groupes auxquels ils appartiennent. Comme mentionné dans la discussion théorique, trois dynamiques relationnelles principales façonnent la chaîne d'interdépendances: la coopération, l'affrontement et la surenchère (outbidding). Ces dernières apparaissent comme le reflet des jeux de pouvoir locaux, dont la teneur jette un flou sur la mise en œuvre des politiques antiterroristes, qui servent bien souvent de paravent aux actions guidées avant tout par des considérations locales. Ces dynamiques relationnelles transcendent tous les ordres de gouvernement. Puisque les dirigeants russes font, depuis l'adoption d'une approche civile, de la restauration des capacités de l'État dans la région un maillon essentiel de leur politique antiterroriste, nous nous penchons d'abord sur les relations qui se nouent entre le fédéral et les entités fédérées, avant de nous tourner vers les interdépendances qui lient les acteurs de terrain de l'antiterrorisme.

\section{Jeux de pouvoir locaux et dynamique de violences}

La lutte antiterroriste est enchâssée dans des pratiques plus larges. Comme R. Sakwa (2011) le montre, l'État russe est un «État duel», caractérisé par la superposition de deux régimes: le régime constitutionnel, régi par la Constitution, et un régime administratif, qui transcende le premier sans en annuler les principes fondateurs. Ce dernier est basé sur l'informalité, qui s'exprime avant tout dans des relations de type clientéliste, qui régissent les interactions entre les différentes factions engagées à différents niveaux dans des luttes de pouvoir. Ce régime administratif est à la fois un «réseau de relations sociales, dans 
lequel les ressources politiques et économiques s'entremêlent [...], et un acteur du processus politique» (Sakwa, 2011, p. 42). Son renforcement depuis l'arrivée de V. Poutine au pouvoir en 2000 n'a fait qu'ancrer plus fermement les valeurs de «hiérarchie, paternalisme et clientélisme» dans la conduite de la politique (Gorenburg, 2012, p. 5). Cependant, l'établissement d'une "verticale du pouvoir» est loin de s'être concrétisée dans les faits. De nombreuses résistances existent sur le plan local, révélant des fractures horizontales, entretenues par l'absence de volonté des acteurs locaux de mettre en œuvre les directives de Moscou, la persistance du localisme et l'omniprésence de la corruption (Monaghan, 2012).

La politique antiterroriste ne fait pas exception à cette règle non écrite. Bien au contraire, le quasi-chaos institutionnel et organisationnel qui règne au Caucase du Nord ne fait qu'accentuer la prévalence du clanisme, du localisme et du clientélisme comme principes organisateurs des relations entre les acteurs impliqués de près ou de loin dans la mise en œuvre des politiques antiterroristes. La nomination des présidents des républiques fédérées par le président de la Fédération, procédure mise en place par les réformes de 2004, prend ici une saveur toute particulière. Il s'agit en effet d'adouber des dirigeants loyaux envers Moscou, mais capables de s'imposer aux clans omnipotents. Ce faisant, Moscou poursuit une politique mise en place lors de la seconde guerre de Tchétchénie, moment fondateur du régime Poutine. L'objectif n'est pas tant de mettre immédiatement fin aux violences, mais de contenir le conflit et de tirer des «bénéfices collatéraux» (Baev, 2004, p. 347; Le Hérou \& Remangey, 2008), qui s'expriment en termes de renforcement du régime et de l'État. Cela passe par des tentatives répétées d'instrumentaliser les jeux locaux afin de maintenir Moscou dans une position dominante.

Le cas du Daghestan est emblématique de l'influence des jeux de pouvoir sur les dynamiques du conflit qui secoue cette république multiethnique et instable. La nomination en janvier 2013 de Ramazan Abdulatipov à sa tête, en remplacement de Magomedsalam Magomedov qui a été remercié deux ans avant la fin de son mandat, a été interprétée comme une nouvelle tentative de Moscou d'installer une personnalité politique proche des réseaux moscovites et capable de mettre de l'ordre dans les querelles entre clans, oligarques et autres potentats locaux (Markedonov, 2013). Cette nomination a entraîné un repositionnement des différents acteurs, ponctué de nouvelles violences 
(Dzutsev, 2011b). Toutefois, Abdulatipov, peu inséré dans les jeux locaux avant son entrée en fonction, se révèle relativement dépourvu de soutiens. Afin de ne pas être marginalisé, il a d'abord nommé G. Makhachev au poste stratégique de vice-premier ministre. Ce dernier, dont l'origine de la fortune est controversée («Dagestan's representative...», 2010), est à la tête d'un clan influent au Daghestan; il possède également de très bons ancrages à Moscou. Puis, Abdulatipov a constitué son administration présidentielle en s'entourant de personnalités recrutées sur les critères de loyauté, eux-mêmes fondés sur l'appartenance à un même groupe ethnique ou à une même région (Magomedov, 2014). Loin de mettre fin à la surenchère entre clans et groupes ethniques, ces nominations n'ont fait que les raviver, perpétuant les pratiques qu'Abdulatipov entendait pourtant combattre.

Lafflux massif de subsides en provenance de Moscou ne fait qu'exacerber un climat de compétitions. Toutefois, le Daghestan n'est pas la seule république aux prises avec ce type de difficultés. De manière générale, les élections ou les changements anticipés à la tête de l'exécutif se traduisent par des montées de violence, qui ne sont, de l'aveu même des principaux intéressés, que le reflet des luttes de pouvoir. Le président de la Kabardino-Balkarie, écarté de son poste en décembre 2013, estime ainsi que l'explosion de violence de 2010 correspond à des tentatives de ses concurrents de démontrer son incapacité à combattre l'insurrection et donc de provoquer sa chute (Dzutsev, 2010a). De telles stratégies passent par la conclusion d'alliances ponctuelles avec des groupes insurgés (International Crisis Group, 2012, p. 80). La violence est ainsi détournée pour satisfaire des intérêts privés et permettre à certains de se maintenir ou de se repositionner dans les luttes de pouvoir quelquefois très localisées. Une telle logique se manifeste, entre autres, par une mise en œuvre à géométrie variable des directives de Moscou, qui se révèle être fonction non pas des objectifs assignés à la lutte antiterroriste ou aux politiques contre-insurrectionnelles, mais découle avant tout d'impératifs immédiats liés à la survie politique d'une personnalité locale, à la préservation ou à l'acquisition de positions de pouvoir pour un groupe, un clan ou une faction locale. 
Entre absence de coordination et compétition: une régionalisation de la lutte anti-terroriste en trompe-l'œil

À ce contexte marqué par une compétition à peine voilée pour l'accès aux ressources symboliques, politiques et matérielles s'ajoute une absence manifeste de coordination entre les différents intervenants. Cette dernière s'explique par la juxtaposition de trois réalités: une forte compartimentalisation bureaucratique et l'absence d'une culture de la concertation; la coexistence de visions et d'intérêts divergents, qui entravent toute velléité de collaboration; et le manque de confiance entre les différents acteurs. Medvedev et Poutine ont reconnu à plusieurs occasions les problèmes que l'absence de coordination pose sur le terrain (Kremlin, 2010a). Ils passent toutefois sous silence les problèmes structurels, tout comme la compétition qui oppose ces mêmes acteurs. La chaîne de commandement militaire reste ainsi déficiente, malgré les réformes lancées après la guerre contre la Géorgie en 2008. À cela s'ajoutent les problèmes posés par la réduction du nombre d'officiers, la vétusté du matériel et le manque de discipline (Khramchikhin, 2010). Parallèlement, Khloponin, qui s'est vu confier une mission de développement économique, n'est doté d'aucun mécanisme de contrôle des actions des forces armées. Ces dernières ne l'informent peu ou pas du lancement d'une opération antiterroriste (Sukhov, 2010), alimentant ainsi la confusion et la compétition inter-agences. Deux visions continuent ainsi à s'affronter sans s'articuler l'une à l'autre: une intervention dominée par une approche civile, axée sur les populations, et une approche favorisant le tout coercitif. Les tensions entre lutte antiterroriste, confiée aux agences et organismes chargés du respect de la loi (police, armée, FSB), et contre-insurrection ont ainsi une résonance toute particulière sur le terrain puisqu'elles consacrent une lutte pour l'accès aux moyens déployés par Moscou.

Cette absence de coordination se manifeste également entre les acteurs fédéraux chargés de redistribuer les subsides alloués par Moscou. En décembre 2012, le ministère des Régions a annoncé qu'une partie des fonds irait à la rénovation ou la construction d'infrastructures sociales. Les ministères des Finances et du Développement économique ont fait immédiatement savoir leur désaccord, alors que Khloponin rappelait qu'aucun arbitrage définitif n'avait été établi (Karpenko, 2012). À cette cacophonie s'ajoute l'existence de tensions locales récurrentes, qui découlent d'intérêts divergents et annihilent toute velléité de coopé- 
ration. Le conflit entre les Ingouches et les Ossètes du Nord au sujet de la région du Prigorodnyj reste latent et est ravivé à chaque épisode violent qui comporte une dimension interethnique, comme l'attentat à la voiture piégée qui a touché en septembre 2010 le marché de Vladikavkaz (Dzutsev, 2010b). L'absence de volonté des présidents ingouche et ossète de négocier de bonne foi ne font qu'exacerber les tensions et annihiler toute volonté de collaboration.

Labsence de coordination avec les acteurs fédéraux et locaux participe d'une même dynamique, mais s'explique également par un manque de confiance envers les polices et milices locales. Ainsi, en 2006, le ministre de l'Intérieur fédéral a mis en place en Ingouchie des «départements de police temporaires», composés de policiers d'origine ethnique russe. Ces derniers agissent parallèlement aux unités policières régulières, composées en majorité d'Ingouches. Des accusations de collusion entre certains membres de ces unités ingouches avec des insurgés ont été invoquées pour justifier la création d'unités qui font double emploi avec les premières et dont l'existence même crée des tensions en Ingouchie (Saradzhyan, 2009). De manière générale, les unités appartenant aux différentes forces armées présentes sur le terrain ne partagent que peu leurs informations (Kramer, 2005, pp. 249-250), agissant en vase clos et rendant illusoire toute régionalisation de la lutte antiterroriste.

\section{Clanisme, criminalisation et détournement de la violence}

Dans un contexte marqué par une asymétrie des relations et la maximisation des intérêts immédiats, les relations entre les représentants de l'État et les autres acteurs en conflit, les insurgés et les réseaux criminalisés, sont loin d'être linéaires et de se résumer à la seule confrontation. Elles comportent deux autres aspects. Tout d'abord, elles peuvent inclure l'échange d'informations, que cette pratique soit le fait de transfuges ou qu'elle soit motivée par une appartenance ethnique, clanique, ou encore par la corruption. De nombreux cas de collusion entre des policiers appartenant aux milices tchétchènes de R. Kadyrov et des insurgés ont ainsi été rapportés (Kramer, 2005, pp. 214-215). Un même constat a été fait dans les autres républiques ("Corruption in North Caucasus...», 2006). Il ne concerne pas les seuls miliciens ou soldats, puisque le chef déchu de la police de Makhachkala a été accusé de connivence avec des groupes insurgés («MVD Dagestana...», 2013). 
Il met en évidence un phénomène de circulation des acteurs d'une catégorie à l'autre.

Deuxièmement, les relations entre dépositaires de la force publique et insurgés peuvent être motivées par des considérations économiques. Le rôle joué par la corruption, l'économie souterraine et les réseaux criminels est ici patent. Le trafic d'armes, les pots-de-vin versés aux membres des forces de l'ordre dans de nombreuses situations (passage aux check-points, négociation de la libération d'un individu arrêté, voire récupération d'un corps...) sont légion (Trenin, Malashenko \& Lieven, 2004, p. 144). De telles pratiques, d'abord cantonnées en Tchétchénie, se sont rapidement étendues à toute la région, contribuant ainsi à une extension des réseaux criminels. P. Baev (2006, p. 81) n'hésite pas à parler dans le cas tchétchène d'une «interpénétration» entre polices et groupes criminels, souvent contrôlés par les insurgés eux-mêmes. De telles interdépendances existent dans les autres républiques, à l'image de ces policiers accusés de vente d'armes aux insurgés en KabardinoBalkarie («Corruption in North Caucasus...», 2006). Ces pratiques incluent également les fonctionnaires des gouvernements locaux, régulièrement mis à contribution par des groupes insurgés pour financer la «guerre sainte» (Rybina \& Sergeev, 2013).

Par ailleurs, la corruption constitue un problème bien plus profond au Caucase du Nord qu'ailleurs, d'après Medvedev lui-même. Il impute cette situation au fait que les réseaux criminalisés se confondent bien souvent avec les clans qui contrôlent l'économie souterraine (Kremlin, 2010c). Cette dernière ne se limite pas aux trafics de matières illicites, mais inclut des biens de consommation courante ou encore l'accès aux services publics comme l'entrée à l'université (sur ce dernier aspect, voir La Cava \& Michael, 2006, p. 10). Dès lors, dans un conflit où les frontières entre catégories d'acteurs sont brouillées par la prédominance du clanisme, la violence est détournée pour disqualifier les rivaux (Baev, 2006, p. 81). Plusieurs opérations menées au nom de la lutte antiterroriste cachent des règlements de compte entre clans rivaux ou anciens alliés, comme l'illustre cette action menée en décembre 2007 dans le village daghestanais de Gimri (RFE/RL, 2008).

Dans un tel contexte, les objectifs généraux des politiques antiterroristes rentrent bien souvent en confrontation directe avec les pratiques des représentants chargés de les mettre en œuvre. Alors que Medvedev appelle au développement d'une approche «compréhensive» qui mette les populations civiles au centre des préoccupations (Kremlin, 2010a), 
les actions menées sur le terrain vont dans un sens tout autre. Ce décalage entre discours et mise en application des décisions prises à Moscou tient à deux dimensions concomitantes: l'absence de discipline dans les rangs des forces armées et des polices locales et le détournement de mesures répressives à des fins privées. Nombre d'abus et de discriminations à l'endroit d'insurgés supposés, de sympathisants ou encore de leurs proches ont été documentés par plusieurs organisations non gouvernementales, montrant par là même leur généralisation (MASHR, 2011; Memorial, 2012). Tout comme lors de la seconde guerre de Tchétchénie, les opérations de ratissage sont souvent ponctuées de vols, de pillage ou de destruction de propriétés. La torture reste très largement pratiquée et il n'est pas rare de voir les hommes suspectés d'être des Salafistes arrêtés et battus dans la rue ou aux check-points (Ratelle, 2012). Dans le même temps, certaines actions punitives sont détournées par ceux chargés de les mettre en place. Parmi les derniers exemples recensés, le président Poutine a proposé en septembre 2013 que soient confisqués les biens des proches des insurgés s'il est prouvé qu'ils ont été acquis grâce à des activités «terroristes» (Khamraev \& Machkin, 2013). Comme le note Dzutsev (2011a), la recherche de preuves est le plus souvent considérée comme une activité superflue par les forces de police. Cette nouvelle mesure risque donc d'alimenter les pratiques prédatrices, institutionnalisées depuis le début de la seconde guerre de Tchétchénie.

De fait, l'impunité qui règne au Caucase du Nord a largement contribué à ancrer dans les comportements le détournement de la violence étatique à des fins privées, et ce, à tous les paliers de gouvernement. Les interdépendances qui lient les acteurs de l'anti-terrorisme sont le plus souvent orientées vers des objectifs à court terme et les gains immédiats plutôt que vers une lutte cohérente contre les insurgés. Loin d'encourager la normalisation des relations entre les dépositaires de la force publique et les populations civiles, ces pratiques contribuent au contraire à la déconnexion grandissante entre autorités locales, fédérales et civiles, et entravent grandement la mise en œuvre des politiques antiterroristes.

\section{Conclusion}

Les maux qui affectent la Russie dans son ensemble trouvent un écho particulièrement alarmant au Caucase du Nord, en proie à de multiples 
conflits. Ces conflits ne se résument pas à un affrontement entre insurgés islamistes et l'État russe. Ils comportent différentes strates et dimensions et relèvent de logiques multiples, qui s'enchâssent les unes dans les autres. Si les initiatives prises par Moscou peuvent sembler louables sur papier, elles se heurtent à la nature même des interactions entre acteurs, dominées par le clientélisme, le localisme et le clanisme. Le décalage entre objectifs déclarés de la lutte antiterroriste et actions sur le terrain devient d'autant plus grand que Moscou peine à s'imposer comme un acteur dominant dans des jeux profondément asymétriques et qui rassemblent une multitude d'acteurs. Moscou se révèle ainsi être un acteur de l'incertitude, au même titre que les acteurs locaux, qui semblent pris dans un jeu dont ils ne maîtrisent pas toujours les tenants et les aboutissants. Ainsi, les règles du jeu contraignent à différents degrés les différents acteurs et permettent la reproduction de certaines pratiques, comme le détournement de la violence dite légitime à des fins privées. La non-reconnaissance des dimensions politiques des conflits qui secouent cette région participent à l'enracinement de ces conflits et à la fragmentation toujours plus poussée d'un territoire profondément divisé autour d'appartenances ethniques, territoriales et religieuses. Ce faisant, la notion de configuration, si elle ne révolutionne pas la compréhension de ce conflit, permet de mieux cerner l'importance des interdépendances qui lient les acteurs de l'antiterrorisme sur les dynamiques du conflit, en tant qu'elle tient compte de leur contingence et met en évidence les contraintes avec lesquelles les acteurs ont à composer.

\section{Références}

Amnesty International. (2012). The Circle of Injustice - A Summary. Security Operations and Human Rights Violations in Ingushetia, Russian Federation. June. EUR 46/005/2012.

Baev, P. (2004). Instrumentalizing Counterterrorism for Regime Consolidation in Putin's Russia. Studies in Conflict and Terrorism, 27(4), 337-352.

Baev, P. (2006). Contre-terrorisme et islamisation du Caucase du Nord. Politique étrangère, 1, 79-89.

Bloom, M. (2004). Palestinian Suicide Bombing: Public Support, Market Share, and Outbidding. Political Science Quarterly, 119(1), 61-88.

Campana, A. (2013a). La régionalisation de la violence au Caucase du Nord. Dans A. Campana \& G. Hervouet (Éds), Terrorisme et insurrection. Évolution des dynamiques conflictuelles et réponses des États (pp. 113-135). Québec: PUQ. 
Campana, A. (2013b). Beyond Norms: The incomplete de-securitization of the Russian Counterterrorism France. Critical Studies on Terrorism, 6(3), 457-472.

Campana, A. (2014). The Multiple Contexts of Russian Counterterrorism Frames: The Framing Process and Discursive Field. Dans D. Pisoiu (Éd.), Arguing Counterterrorism New Perspectives (pp. 322-350). London: Routledge.

Campana, A., \& Légaré, K. (2011). Russia's Counterterrorism Operation in Chechnya: Institutional Competition and Issue Frames. Studies in Conflict and Terrorism, 34(1), 47-63.

Campana, A., \& Ratelle, J.-F. (2014). A Political Sociology Approach to the Diffusion of Conflict from Chechnya to Dagestan and Ingushetia. Studies in Conflict and Terrorism, 37(2), 115-134.

Derluguian, G. (2005). Bourdieu's Secret Admirer in the Caucasus. A World-System Biography. Chicago, IL: The University of Chicago Press.

Devotta, N. (2005). From ethnic outbidding to ethnic conflict: the institutional bases for Sri Lanka's separatist war. Nations and Nationalism, 11(1), 141-159.

Dzutsev, V. (2010a). Security in Kabardino-Balkaria Fails to Improve After Reappointment of President. Eurasia Daily Monitor, 7(165). Consulté le 25 septembre 2013. Repéré à http://www.jamestown.org/programs/edm/ single/?tx_ttnews\%5Btt_news\%5D=36849\&tx_ttnews\%5BbackPid\%5D $=484 \&$ no_cache $=1$

Dzutsev, V. (2010b). Threat of Renewed Ethnic Clashes Reemerges Following North Ossetia Suicide Attack. Eurasia Daily Monitor, 7(168). Consulté le 6 octobre 2011. Repéré à http://www.jamestown.org/programs/edm/ single/?tx_ttnews\%5Btt_news\%5D=36883\&tx_ttnews\%5BbackPid\%5D $=484 \&$ no_cache $=1$.

Dzutsev, V. (2011a). Dagestan Dubbed the Most Dangerous Place in the North Caucasus. Eurasia Daily Monitor, 8(183). Consulté le 6 octobre 2011. Repéré à http://www.jamestown.org/programs/edm/single/?tx_ttnews\%5Btt_ news $\% 5 \mathrm{D}=38492 \& \mathrm{cHash}=3180 \mathrm{f} 8 \mathrm{c07d} 15 \mathrm{ec} 1 \mathrm{e} 93 \mathrm{e} 3 \mathrm{e} 2 \mathrm{ba} 9 \mathrm{a} 8 \mathrm{e} 4840$.

Dzutsev, V. (2011b). So Far, New Dagestani President's Reforms Appear to Bring More Violence. Eurasia Daily Monitor, 10(173). Consulté le 1 octobre 2013. Repéré à http://www.jamestown.org/programs/edm/single/?tx_ttnews\% 5Btt_news $\% 5 \mathrm{D}=41424 \& \mathrm{tx} \_\mathrm{ttnews} \% 5 \mathrm{BbackPid} \% 5 \mathrm{D}=27 \& \mathrm{cHash}=2 \mathrm{fe} 00 \mathrm{f}$ 5c735d531fb7507a33aa5db9f1

Elias, N. (1978). What is Sociology? (traduction de S. Mennel et G.Morrissey). New York, NY: Columbia University Press.

Elias, N., \& Dunning, E. (1986). Quest for Excitement: Sport and Leisure in the Civilizing Process. New York, NY: B. Blackwell.

Emirbayer, M. (1997). Manifesto for a Relational Sociology. American Journal of Sociology, 103(2), 281-317.

Galeotti, M. (2002). 'Brotherhoods' and 'Associates': Chechen Networks of Crime and Resistance. Low Intensity Conflict $\mathfrak{F}$ Law Enforcement, 11(2-3), 340-352.

Gilligan, E. (2009). Terror in Chechnya: Russia and the Tragedy of Civilians in War. Princeton, NJ: Princeton University Press. 
Gorenburg, D. (2012). The Rules of the Political Game in Russia. Russian Politics and Law, 50(3), 3-6.

Hahn, G. (2011). Getting the Caucasus Emirate Right. Washington: CSIS Russia and Eurasia Program. Consulté le 15 février 2013. Repéré à http://csis.org/ files/publication/110930_Hahn_GettingCaucasusEmirateRt_Web.pdf.

Horowitz, D. (1985). Ethnic Groups in Conflict. Berkeley, CA: University of California Press.

International Crisis Group. (2012). The North Caucasus: the Challenges of Integration (II), Islam, the Insurgency and Counter-Insurgency. Europe Report $\mathrm{N}^{\circ} 221$.

Kalyvas, S. (2003). The Ontology of Political Violence: Action and Identity in Civil Wars. Perspectives on Politics, 1(3), 475-494.

Kalyvas, S. (2007). Civil wars. Dans C. Boix \& S. C. Stokes (Éds.). The Oxford handbook of Comparative Politics (pp. 416-439). Oxford: Oxford University Press.

Kalyvas, S. (2008). Promises and pitfalls of an emerging research program: the microdynamics of civil war. Dans S. Kalyvas, I. Shapiro \& T. Masoud (Éds.), Order, conflict and violence (pp. 397-421). Cambridge: Cambridge University Press.

Kalyvas, S. (2010). Foreword Internal Conflict and Political Violence: New Developments in Research. Dans E. Chenoweth \& A. Lawrence (Éds.), Rethinking Violence. State and Non-State Actors in Conflict (pp. xi-xiii). Cambridge, MA: MIT Press.

Karpenko, E. (2012, 13décembre). Krisis spisal Kavkaz. Gazeta.ru. Consulté le 25 septembre 2013. Repéré à http://www.gazeta.ru/business/2012/12/ 13/4890173.shtml.

Khamraev, V., \& Machkin, S. (2013, 28 septembre). Za Terrorista rasplatâtcâ te, kto emu dorog. Kommersant.ru. Consulté le 30 septembre 2013. Repéré à http://kommersant.ru/doc/2307965?fb_ref=fb.

Khramchikhin, A. (2010, 13 août). Uroki "pâtidnevnoj vojny". Nezavisimoye Voyennoye Obozreniye. Consulté le 6 octobre 2011. Repéré à http://nvo. ng.ru/wars/2010-08-13/1_5d_war.html.

Kramer, M. (2005). Guerrilla Warfare, Counterinsurgency and Terrorism in the North Caucasus: The Military Dimension of the Russian-Chechen Conflict. Europe-Asia Studies, 57(2), 209-290.

Kremlin. (2010a). Neobhodimo raš̌irim' i užestoč it' mery po bor'be sterrorismom. Consulté le 12 août 2011. Repéré à http://kremlin.ru/news/7307.

Kremlin. (2010b, 27 février). Opening Remarks at Meeting on Security in the North Caucasus Federal District. Nalchik. Consulté le 11 août 2011. Repéré à http:// archive.kremlin.ru/eng/text/speeches/2010/02/27/1800_type82913_ 224525.shtml.

Kryshtanovskaya, O. (2012). Formats of Russian State Power. Russian Politics and $L a w, 50(3), 7-17$.

La Cava, G., \& Michael, S. (2006). Youth in the North Caucasus: From Risks to Opportunity. Washington, DC: The World Bank. 
Le Huérou, A., \& Regamey, A. (2008). La guerre russe en Tchétchénie : discours antiterroriste et légitimation de la violence. Critique internationale 4(41), 99-118.

Magmedov, R. (2014, 9 janvier). Glava Dagestana i tlâratincy. Zemlâki i radstvenniki Ramazana Abdulatipova v vysših ešelonah vlasti. Kazkavskaâ Politika. Consulté le 12 janvier 2014. Repéré à http://kavpolit.com/glavadagestana-i-tlyaratincy/.

Malashenko, A., \& Yarlykapov, A. (2009). Radicalisation of Russia's Muslim Community. MICROCON Policy Working Paper 9. Brighton: MICROCON.

Markedonov, S. (2010, 1er novembre). A Strategy for North Caucasus: Don't Mention Politics or Religion! Od-Russia, Post-Soviet World, Open Democracy. Consulté le 21 octobre 2012. Repéré à http://www.opendemocracy.net/ od-russia/sergei-markedonov/strategy-for-north-caucasus-don't-mentionpolitics-or-religion.

Markedonov, S. (2013, 1er février). Sizing up Dagestan's newly appointed leader. Russia Beyond the Headlines. Consulté le 25 septembre 2013. Repéré à http://rbth.ru/opinion/2013/02/01/sizing_up_dagestans_newly_ appointed_leader_22409.html.

MASHR (2011). Bez Prava na Žizn'. 2011 god. Respublika Ingušetiâ. Nazran. Consulté le 12 mars 2013. Repéré à http://www.mashr.org/?page_id=9.

Melvin, N. (2007). Building Stability in the North Caucasus Ways Forward for Russia and the European Union. SIPRI Policy Paper, 16.

Memorial. (2011). Novyj kurs Magomedova? Situaciâ s prabami čelaveka I popytky konsolidacii obšestva Respubliki Dagestana, Mars 2010 - Mars 2011, Moscou. Consulté le 12 mars 2013. Repéré à http://www.memo.ru/d/2574.html.

Monaghan, A. (2012). The vertikal: power and authority in Russia. International Affairs 88(1), 1-16.

Moore C., \& Tumelty, P. (2008). Foreign Fighters and the Case of Chechnya: A Critical Assessment. Studies in Conflict and Terrorism, 31(5), 412-433.

O'Loughlin, J., \& Witmer, F. (2011). The Localized Geographies of Violence in the North Caucasus of Russia, 1999-2007. Annals. Association of American Geographers, 101(1), 178-201.

Raleigh, C. (2011). Scales of Conflict Research. International Interactions: Empirical and Theoretical Research in International Relations, 37(4), 469-481.

Ratelle, J.-F. (2012). Radical Islam and the Chechen War Spillover: A Political Ethnographic Reassessment of the Upsurge of Violence in the North Caucasus since 2009 (these de doctorat non publiée). Ottawa University.

RFE/RL (2008, 7 janvier). RFE/RF Newslines. RFE/RL. Consulté le 12 mars 2010. Repéré à http://www.rferl.org/content/article/1144025.html.

Russell, J. (2008). Ramzan Kadyrov: the indigenous key to success in Putin's Chechenization Strategy? Nationalities Papers, 36(4), 659-687.

Russell, J. (2010). Case Study: Ramzan Kadyrov in Chechnya: Authoritarian Leadership in the Caucasus. Dans D. Canter (Éd.), The Faces of terrorism: multidisciplinary perspectives (pp. 209-226). Chichester: Wiley-Blackwell. 
Rybina, Û., \& Sergeev, N. (2013, 21 août). Bujnakskih voebikov zastali doma. Kommersant.ru. Consulté le 25 septembre 2013. Repéré à http://kommersant.ru/ $\mathrm{doc} / 2259730$.

Sakwa, R. (2005). Chechnya: From Past to Future. London: Anthem Press.

Sakwa, R. (2011). The Crisis of Russian Democracy: The Dual State, Factionalism and the Medvedev Succession. Cambridge: Cambridge University Press.

Saradzhyan, S. (2009, 24 août). Ingushetia: Counterterrorism Failure. International Relations and Security Network. Consulté le 6 octobre 2011. Repéré à http://www.isn.ethz.ch/Digital-Library/Articles/Detail//?ots591 $=$ EB06339B-2726-928E-0216-1B3F15392DD8 \& coguid $=9$ C 879 A60 8A40-14E8-76C3-2C016AE9096C\&lng=en \&id=105020

Schaefer, R. W. (2011). The Insurgency in Chechnya and North Caucasus: From Gazavat to Jihad. Santa Barbara, CA: Praeger Security International.

Schwartzbaum A. (2013). Dagestan's Hot Summer. Russia and Eurasia Program's (REP) Blog. Center for Strategic and International Studies. Consulté le 10 septembre 2013. Repéré à http://csis.org/blog/dagestans-hot-summer.

Shterin, M., \& Yarlykapov, A. (2011). Reconsidering Radicalisation and Terrorism: the New Muslims Movement in Kabardino-Balkaria an Its Path to violence. Religion, State, and Society, 39(2-3), 303-325.

Soeters, J. L. (2005). Ethnic Conflict and Terrorism. The Origins and Dynamics of Civil War. New York, NY: Routledge.

Sokolov, D., Magomedov H., \& Silaev, N. (2013). Istočniki konfliktov I razvitiâ na Cevernom Kavkaze, Doklad Kavkazskogo centra proektnyh rešenij. Consulté le 23 septembre 2013. Repéré à http://www.kavkaz-uzel.ru/ articles/222451/.

Soldatov, A., \& Borogan, I. (2010). The New Nobility. The Restoration of Russia's Security State and the Enduring Legacy of the KGB. New York, NY: PublicAffairs.

Sukhov, I. (2010, 3 septembre). War on Terror: the North Caucasus Federal District. Argentura.ru. Consulté le 6 octobre 2011. Repéré à http://www. agentura.ru/english/terrorism/ncfd/.

Tilly, C. (2005). Terror as Strategy and Relational Process. International Journal of Comparative Sociology, 46(1-2), 11-32.

Tishkov, V. (2004). Chechnya: Life in a War-Torn Society. Berkeley, CA: University of California Press.

Toft, M., \& Zhukov, Y. (2012). Denial and punishment in the North Caucasus. Evaluating the effectiveness of coercive counter-insurgency. Journal of Peace Research, 49(6) 785-800.

Trenin, V., Malashenko, A., \& Lieven, A. (2004). Russia's Restless Frontier. The Chechnya Factor in Post-Soviet Russia. Washington, DC: Carnegie Endowment for the International Peace.

Vatchagaev, M. (2011). Ingush in 2010: The Insurgency Remains a Potent Force. North Caucasus Analysis, 12(2). Consulté le 1er février 2011. Repéré à http://www.jamestown.org/single/?no_cache $=1 \&$ tx_ttnews $\% 5 \mathrm{Btt}$ news $\% 5 \mathrm{D}=37416 \&$ tx_ttnews $\% 5 \mathrm{BbackPid} \% 5 \mathrm{D}=514$. 
Wood, T. (2007). Chechnya: The Case for Independence. London: Verso.

\section{Documents en ligne}

Corruption in the North Caucasus Fuels Instability. (2006, 30 novembre). North Caucasus Weekly, 7(46). Consulté le 12 mars 2010. Repéré à http://www. jamestown.org/programs/nc/single/?tx_ttnews\%5Btt_news $\% 5 \mathrm{D}=$ 32283\&tx_ttnews\%5BbackPid\%5D $=188 \&$ no_cache $=1$

Dagestan's representative at Russian President Makhachev refutes WikiLeaks on his luxury houses. (2010, 29 novembre) Caucasian Knot. Consulté le 25 septembre 2013. Repéré à http://eng.kavkaz-uzel.ru/articles/15368/.

Korrupciâ na Severnom Kavkaze ugrožaet nasbezopasnosti-Medvedev. (2010, 19 mai). Ria-novosti.ru. Consulté le 12 août 2011. Repéré à http://ria.ru/ video/20100519/236180881.html.

MVD Dagestana podverdilo otstranenie ot dolžnosti nacal'nika poličii. (2013, 26 mars). Kavkaz Online. Consulté le 25 septembre 2013. Repéré à http:// kavkasia.net/Russia/2013/1364325871.php.

V Kabardino-Balkarii vozobnovlâetsâ operaciâ 'Oružie'. (2010, 7 mars). Kavkazskij Uzel. Consulté le 15 juin 2010. Repéré à http://47.kavkaz-uzel.ru/ articles/150633.

ABSTRACT - This article analyzes the dynamics of the Russian counter-terrorism policies in the North Caucasus and their effects on the conflict. Relying on Elias ${ }^{1}$ concept of configuration, it deconstructs the interdependencies between the main actors involved in counter-terrorism and examines their nature and logic. It shows that there is not only a wide gap between discourses and actions implemented on the ground but also that the main counter-terrorist actors do not have the same interests and beliefs. The predominance of clanism, localism and clientelism as the main modes of interactions and organizational principles only strengthens the unwillingness of actors to work together. In so doing, it explains the failure of the counter-terrorism policies by focusing on the power games, which are taking place in Moscow as well in the North Caucasus; the absence of coordination; the inter-agency competition and the use of violence to meet private interests. It demonstrates that such practices fuel the upsurge of violence and nurture a conflict, which follows multiple logics.

KEY WORDS • Russia, North Caucasus, Anti-terrorist policies, networks, interdependence.

RESUMEN - El presente artículo trata sobre las dinámicas propias a las políticas rusas antiterroristas en el Cáucaso Norte, y analiza sus impactos sobre el conflicto. Para ello, se basa sobre el concepto de configuración tal como lo desarrolla N. Elias. El artículo se propone deconstruir las interdependencias que unen los actores del antiterrorismo y examinar su naturaleza y sus lógicas. Se muestra que existe, no sólo una distancia entre los discursos y las prácticas, sino también una divergencia de intereses y de creencias, que el predominio del clan, del localismo y del clientelismo, como modos de interacciones y de principios organizacionales, no hace que enraizar. El texto explica el fracaso de las políticas antiterroristas por los juegos de poder que, en Moscú y en el Norte del Cáucaso, dificultan su buena implementación por la ausencia de coordina- 
ción, por la competencia entre agencias, y por la utilización de la violencia a fines privados. Queda demostrado así que, lejos de contener el conflicto, éstas prácticas, imputables a diferentes actores implicados en la lucha antiterrorista, participan en el incremento de las violencias y mantienen un conflicto de lógicas múltiples.

PALABRAS-CLAVE - Rusia, Cáucaso del Norte, políticas antiterroristas, redes, interdependencias. 